\title{
miR-489 Confines Uncontrolled Estrogen Signaling \\ Through a Negative Feedback Mechanism and Regulates Tamoxifen Resistance in Breast Cancer
}

\section{Mithil Soni}

University of South Carolina

Ozge Saatci

University of South Carolina

Gourab Gupta

University of South Carolina

Yogin Patel

University of South Carolina

Manikanda Raja Keerthi Raja

University of South Carolina

Jie Li

University of South Carolina

Xinfeng Liu

University of South Carolina

Peisheng Xu

University of South Carolina

Hongjun Wang

Stevens Institute of Technology

Daping Fan

University of South Carolina

Ozgur Sahin

University of South Carolina

Hexin Chen ( $\nabla$ hchen@biol.sc.edu )

University of South Carolina

\section{Research Article}

Keywords: miR-489, breast cancer, estrogen receptor, tamoxifen resistance, CRISPR/Cas9

Posted Date: January 5th, 2022

DOl: https://doi.org/10.21203/rs.3.rs-131460/v2 
License: (c) (i) This work is licensed under a Creative Commons Attribution 4.0 International License. Read Full License 


\section{Abstract}

Background: Approximately $75 \%$ of diagnosed breast cancer tumors are estrogen-receptor (ER) positive tumors and are associated with better prognosis due to their response to hormonal therapies. However, around $40 \%$ of patients relapse after hormonal therapies. In the current study, we aim to evaluate miR-489 as a novel molecular target to combat tamoxifen resistance.

Methods: Genomic analysis of gene expression profiles in primary breast cancers and tamoxifen resistant cell lines unveiled the potential role of miR-489 in regulation of estrogen signaling and development of tamoxifen resistance. We manipulated miR-489 expression in breast cancer cell lines by transient transfection of a miR-489 mimic or establishment of knockout cell lines using the CRISPR/Cas9 system to study the reciprocal regulation of miR-489 and estrogen/ER signaling pathways. Cell proliferation assays, Sphere-formation assays and flow cytometry analysis were conducted to investigate the role of miR-489 in estrogen-induced cell proliferation, cancer stem cell expansion and development of tamoxifen resistance.

Results: miR-489 expression was significantly downregulated in tamoxifen-resistant cell lines. Low levels of miR-489 were associated with poor clinical outcomes in patients with hormone treatment. In vitro analysis showed that loss of miR-489 expression promoted tamoxifen resistance while overexpression of miR-489 in tamoxifen-resistant cells restored tamoxifen sensitivity. Mechanistically, we found that miR489 is an estrogen regulated miRNA that negatively regulated estrogen receptor signaling by using at least the following two mechanisms: i) modulation of ER phosphorylation status by inhibiting MAPK and AKT kinase activities and downregulating SHP2 expression; ii) regulation of nucleus to cytosol translocation of estrogen receptor a (ERa) by decreasing p38 expression and consequently ER phosphorylation. In addition, miR-489 could break the positive feed-forward loop between the estrogenERa axis and p38 MAPK in breast cancer cells, which was necessary for its function as a transcription factor.

Conclusion: Our study unveiled the underlying molecular mechanism by which miR-489 regulates estrogen signaling pathway through a negative feedback loop and uncovered its role in both the development of and overcoming of tamoxifen resistance in breast cancers.

\section{Introduction}

Oncogenic activation of the estrogen receptor (ER) signaling pathway occurs in over $70 \%$ of breast cancers(1). Although, this subtype of breast cancer has the best prognosis due to targeted endocrine therapies, most patients with advanced disease eventually develop resistance to these endocrine therapies. Even for patients treated in the adjuvant setting, a considerable risk of relapse persists indefinitely(2). Furthermore, approximate $50 \%$ of patients with locally advanced or metastatic ER+ breast cancer do not respond to first-line endocrine treatment(3).Additionally, most patients who initially respond to the therapy eventually develop acquired resistance(4). Despite significant research efforts and 
discoveries made in recent years, the exact reasons for endocrine therapy failure in patients with ER+ breast cancer remain largely unknown. Published studies have implicated the mutations in the ESR1 gene; epigenetic silencing of ESR1, activated growth factor receptor signaling, including the EGFR/HER2 pathway, the PI3K-AKT pathway and the MAPK pathway; and overexpression of co-activators such as NCOA3 and FOXA1, as important mechanisms of de novo or acquired resistance $(5,6)$. However, the only mechanisms of antiestrogen resistance that are supported in the clinic are HER2 amplification, mutations in the ligand-binding domain (LBD) of ESR and dysregulation of the CDK4/6 pathway(7-9). Discovery of novel agents that simultaneously modulate these pathways may help development of improved targeted combination strategies to combat endocrine resistance.

Dysregulation of miRNAs has been increasingly recognized as a critical contributor to cancer development, progression, and therapy resistance. Many miRNAs have been reported to contribute to endocrine resistance. Downregulation of miR-489 has been observed in tamoxifen-resistant breast cancer, but its functional involvement remains unexplored $(10,11)$. In this study, we systematically investigated the functional roles of miR-489 in ER+ breast cancer. We demonstrated that downregulated miR-489 expression significantly stimulates estrogen dependent and independent growth and also promoted tamoxifen resistance through hyper activation of E2-ERa, HER2-PI3K-AKT and ERK signaling pathways. Thus, patients with ER+ breast cancer together with low miR-489 expression may be intrinsically resistant to endocrine therapies.

\section{Materials And Methods}

The detailed procedures of cell culture, antibody and immunoblot, flow cytometry, qRT-PCR, cytoplasmic and nuclear fractionation, luciferase reporter assay, microarray, tumorsphere formation assay, colony formation assay, CRISPR/Cas9-mediated genomic editing, and immunochemical staining, are described in Supplemental Experimental Procedures (Additional file 1).

\section{Cell lines and culture}

MCF7, T47D, and HCC1954 were purchased from ATCC in 2013. MDA-MB231, MDA-MB-468, MDAMB361, Hs578T, ZR-75-1, and BT474 cells were obtained from Dr. Saraswati Sukuma (Johns Hopkins University), in 2008. MCF7 vector and MCF7 HER2 cell lines were kindly provided by Dr. Rachel Schiff (Baylor College of Medicine). MCF7-WT and tamoxifen resistant MCF7-TAMR cells were established as previously reported (12). Cells were grown under standard conditions(13-15).

\section{Tamoxifen-sensitization assays}

Tamoxifen resistant cell lines were treated with control siRNA or miR-489 mimic with or without tamoxifen at indicated concentrations for 72 hours. Similarly, tamoxifen-sensitive cell lines were treated with a control siRNA or a miR-489 inhibitor with or without tamoxifen at indicated concentrations for 72 hours. MTT based cell viability assays and colony formation assays were carried out to examine tamoxifen sensitization. 


\section{Generation of knock out cells}

CRISPR/Cas9 gene editing method was used to generate the miR-489 knock out cell line as shown in our previous publication(15). Two guide RNAs flanking pre-miR-489 were designed using guide RNA designing tool (cripsr.mit.edu). G-block guide RNAs were purchased from IDT technologies; Cas9-GFP plasmid was obtained from Dr. Philip Buckhaults. Gblock guide RNA and Cas9-GFP were co-transfected in T47D cells using T47D avalanche transfection reagent (ez biosystem). 72 hours post transfection, GFP positive cells were sorted using a Fluorescence activated cell sorter. Sorted cells were then diluted to single cells and seeded into a 96-well plate. The rest of the cells were seeded onto a $10-\mathrm{cm}$ dish. Colonies grown from individual clones were then expanded and screened for miR-489 deletion using genotyping.

\section{Statistical analyses}

Statistical analyses were conducted with $\mathrm{R}$ and GraphPad software packages. A Student t-test or ANOVA test was used for comparison of quantitative data. Gene expression profiles of miR-489, its host gene calcitonin receptor, estrogen receptor alpha, and progesterone receptor were evaluated using a published data set containing 1302 breast cancer patients $(38,39)$ that were stratified by the mean value of miR-489 expression levels. The linear correlations between miR-489 and CALCR, ER- $\alpha$ and PGR genes expression in primary breast cancer tissues were evaluated with the Pearson correlation coefficient analysis. Values of $p<0.05$ were considered statistically significant.

miR-489 signature was generated by using the most up or downregulated genes $(-2<\mathrm{FC}<2, \mathrm{n}=304)$ upon miR-489 overexpression. Expression of the signature genes in patients was converted into z-scores. To calculate a miR-489 signature score for each patient, sum of z-scores of downregulated genes was subtracted from the sum of z-scores of up regulated genes (1). PI3K_ERBB2, p38/MAPK, and estradiol responsive gene signatures (https://reactome.org) in patients were generated by summing up the zscores of the signature genes for each patient (1). Significance for the survival analysis was calculated following a Log-Rank test.

\section{Results}

\section{miR-489 expression is lost in tamoxifen resistance, predicts breast cancer aggressiveness and is regulated by estrogen/ERa axis}

To specifically identify miRNAs that are clinically relevant in endocrine resistance, we analyzed miRNA screening datasets of endocrine resistant models previously published by three independent laboratories including our own (Fig. 1A)(11,12,16). Since all three models are derived through completely different processes, they represent independent tamoxifen resistant models with different resistance mechanisms. The MCF7-HER2 cell line acquired resistance through the activation of the HER2 oncogenic pathway while MCF7-TAM and MCF7:2A represent acquired resistance through long-term culture in tamoxifencontaining and estrogen-deprived media respectively. miRNAs that are dysregulated in all three cell lines suggest their potential roles in the regulation of multiple mechanisms involved in tamoxifen resistance, 
which may therefore be more clinically relevant with promising therapeutic applicationto address tamoxifen resistance. Although many miRNAs were dysregulated in these cell lines, we only found few miRNAs that were aberrantly expressed in all three cell lines (Fig. 1B). Out of these miRNAs, miR-135b, miR-33b, and miR-505 showed an opposite expression pattern among these cell lines. miR-378a-3p and miR-218 were significantly upregulated in all three cell lines while miR-342-5p and miR-489 were significantly downregulated. Intriguingly, miR-489 was one of the top downregulated miRNAs in all three datasets, suggesting its role in tamoxifen resistance. We validated these results using qRT-PCR and indeed found significant downregulation of miR-489 in both resistant cell lines (Fig. 1C). To determine whether the expression of miR-489 was associated with endocrine resistance in patient cohorts, we examined miR-489 expression in hormone-therapy treated ER+ breast cancer patients. We observed a statistically significant association between lower miR-489 expression and poorer overall survival in these patients. In fact, miR-489 expression remained an independent prognostic factor in hormone-therapy treated breast cancer patients obtained from two independent datasets, GSE19783 and METBRIC (Fig. 1D). These results suggest that the loss of miR-489 may promote tamoxifen resistance.

Previously we observed that the average expression of miR-489 was notably higher in luminal cells compared to basal cells $(13,16)$. Furthermore, analysis of miR-489 expression on 13 different breast cancer cell lines also demonstrated that it was expressed at a higher level in hormone positive luminal breast cancer cell lines (Fig. 1E) compared to the cell lines from other subtypes. miR-489 is an intragenic microRNA located in the intron region of CALCR. Analysis of primary breast tumors revealed that miR-489 expression positively correlated with the expression of CALCR, ESR1 and ER responsive genes such as PGR (Fig. 1F), suggesting that miR-489 expression may be regulated by estrogen signaling. To examine this hypothesis, we stimulated three ER+ breast cancer cell lines (T47D, MCF7, and BT474) with estrogen or ethanol for indicated time periods and measured the expression of miR-489 and its host gene CALCR. We found significant upregulation of miR-489 and CALCR in all three cell lines treated with estrogen (Fig. $1 G)$. Estrogen regulation of miR-489 was further investigated in complete media or estrogen deprived media. As expected, depletion of estrogen drastically reduced the expression of miR-489 and CALCR like other classical ER target genes such as trefoil factor 1 (TFF1), progesterone receptor (PGR), and C-X-C motif chemokine ligand 12 (CXCL12) (Fig. 1H). In summary, this data strongly suggests that miR-489 is an estrogen regulated miRNA in breast cancer and may play a regulatory role in tamoxifen resistance.

\section{miR-489 restoration overcomes tamoxifen resistance.}

Since miR-489 was lost in tamoxifen resistant tumors and cell lines, we asked whether restoration of miR489 would sensitize the resistant cell lines. Our previous studies have shown that overexpression of miR489 inhibited breast cancer cells proliferation $(13,16)$. We first tested whether Tamoxifen-resistant cells were still sensitive to miR-489 mimics. Notably, we observed that tamoxifen resistant cell lines, MCF7TAM and MCF-HER2, were just as sensitive to miR-489 mimics as their sensitive counterparts, as opposed to their different sensitivities to tamoxifen (Fig. 2A, 2B). These results further bolstered the possibility that miR-489 might target pathways involved in resistance and could potentially sensitize these resistant cell lines to tamoxifen. Indeed, forced expression of miR-489 significantly sensitized both resistant cell lines 
to tamoxifen. Tamoxifen alone had no significant effect on the growth of both resistant cell lines at $5 \mu \mathrm{M}$, while combination with miR-489 led to around 40\% growth inhibition of MCF7-TAM (Fig. 2C) and 30\% growth inhibition in MCF7-HER2 cells (Fig. 2D). To determine whether miR-489 suppresses cell growth independently or has a synergy effect with tamoxifen, we performed a synergy analysis. In MCF7-TAM cells, the combination of miR-489 and tamoxifen achieved synergistic effects at high levels (fraction reduction >0.2). However, in MCF7-HER2 cells, this combination showed a slight synergy (nearly additive effect) at most levels (Fig. S1A and 1B, additional file 2). In both cell lines, this combination could dramatically reduce the dose of either miR-489 or tamoxifen required to achieve the same level of growth inhibition. These results indicated that miR-489 and tamoxifen can synergistically inhibit cell growth in a cell-line dependent manner.

To further assess the role of miR-489 in tamoxifen resistance, we inhibited endogenous miR-489 in tamoxifen sensitive cells MCF7-Vec and MCF7-WT cells. As expected, inhibition of miR-489 significantly increased tamoxifen resistance in both sensitive cell lines. At the highest concentration tested, inhibition of miR-489 increased survival by $25 \%$ and $40 \%$ in sensitive counterpart of MCF7-Vec and MCF7-WT, respectively (Fig. 2E, 2F). Similarly, colony formation assays also revealed that forced expression of miR489 significantly reduced the survival and colony forming ability in resistant cell lines, while inhibition of endogenous miR-489 enhanced the survival of sensitive counterpart and promoted tamoxifen resistance. (Fig. 2G). To rule out the possibility of the off-target effect of the miR-489 inhibitor, we utilized CRISPR/ Cas9 gene editing to create a miR-489 knockout cell line (Additional file, Fig. S2A). We validated these knock out cells using genotyping and sequencing to ensure the deletion of miR-489. As expected, miR-89 knockout cells exhibited increased growth rate (Additional file, Fig. S2B-2C). Consistently, miR-489 knockout cells also showed significant resistance to tamoxifen, evidenced by MTT based cell viability and colony formation assays (Fig. $2 \mathrm{H}$ and 2l). These results provide direct evidence to support that loss of miR-489 contributes to development of tamoxifen resistance.

\section{miR-489 acts as an endogenous negative feedback regulator to balance estrogen signaling}

To elucidate the underlying pathways targeted by miR-489 to induced tamoxifen sensitization, we reexamined the gene expression profiles of T47D cells transfected with miR-489 mimics and scrambled RNA(13). Interestingly, gene expression analysis revealed enrichment of multiple pathways involved in estrogen signaling and tamoxifen resistance (Fig 3A, 3B). We observed enrichment of ErbB signaling pathway and several stress associated pathways including endoplasmic reticulum (ER) stress and lysosomal pathways. All of these pathways have been previously reported to be involved in tamoxifen resistance $(4,9,17-21)$. Meanwhile, these results are also in accordance with our previous studies showing the role of miR-489 in HER2 signaling, metabolic stress, and autophagy regulation $(13,14,16)$.

Interestingly, we noticed significant enrichment of estrogen-dependent gene expression and ESRmediated signaling. Upon further analysis, we found substantial downregulation of estrogen responsive genes (Fig. 3C). This data suggested that estrogen regulated miR-489 might function as a negative regulator of estrogen signaling. To determine the clinical significance of this data, we applied a miR-489 gene expression signature to a gene expression profile obtained from patient datasets. Consistent with 
our microarray results, we found a strong inverse correlation between the estrogen responsive gene signature and the miR-489 signature and between the PI3K-ERBB2 signature and the miR-489 signature (Additional file 2, Fig. S3A-B). In addition, we noticed that low miR-489 expression in ER+ breast cancer was indicative of worse overall survival (Additional file 2, Fig. 3C), further supporting an essential tumor suppressive role of miR-489 in ER+ breast cancer.

To examine how miR-489 negatively regulated estrogen signaling, we measured transcriptional activity of estrogen receptors by performing a Luciferase reporter assay with a T47D-ERE-Luc reporter cell line. We observed the inhibition of estrogen receptor transcriptional activity upon miR-489 overexpression and increased activity upon inhibition of endogenous miR-489 (Fig. 3D). Consistently, gene expression levels of estrogen responsive genes were further increased upon estrogen stimulation in knock out cells compared to wild type cells (Fig. 3E). We then validated the microarray results by performing qRT-PCR analysis on ER+ and ER-cell lines. As expected, miR-489 caused down regulation of estrogen responsive genes only in ER+ cell lines including T47D, MCF7, and BT474 cells, but did not affect or in some instances increased the expression of these genes in ER- cell lines such as AU565 and HCT116 cells (Fig. $3 F-G)$. These results suggest that miR-489 regulates the expression of these ERa-downstream genes by inhibiting estrogen signaling.

\section{miR-489 inhibits ER-induced cell proliferation and cancer stem cells expansion}

Our previous studies have shown that miR-489 inhibits proliferation of all breast cancer cell lines including ERa+ cell lines $(13,16)$. However, it remains unknown whether miR489-mediated growth inhibition in ER+ breast cancer cells is due to its effects on ER signaling. To investigate this, we examined the effects of miR-489 on estrogen-induced cell proliferation in MCF7 and T47D cells. Both cell lines showed poor proliferation when treated with the vehicle (ethanol), while estrogen treatment enhanced proliferation by more than 2-fold and 4-fold in MCF7 and T47D cell lines respectively. Restoration of miR489 completely abolished estrogen-induced proliferation in both cell lines while inhibition of endogenous miR-489 further increased estrogen-induced proliferation by more than 2 fold in MCF7 and more than 3 fold in T47D cell lines (Fig. 4A-B). Simar patterns were observed in colony formation assay by modulating miR-489 expression in MCF7 and T47D cells (Fig. 4C-D). Forced expression of miR-489 almost completely inhibited estrogen induced colony formation of both cells. Interestingly, inhibition of endogenous miR-489 drastically enhanced estrogen-mediated colony formation (Fig. 4C).

Estrogen treatment has been previously shown to enhance the population of stem-like cells in ERa cell lines(22). These so-called cancer stem cells are thought to be responsible for tumor relapse(23). We hypothesized that miR-489 may inhibit estrogen-induced population of cancerous stem-like cells by its effect on estrogen signaling. Therefore, we studied the effect of miR-489 on the estrogen-induced cancerous stem cell population using colony formation assay, mammosphere assay and flow cytometry. Consistent with previous results, estrogen increased the cancerous stem-like cell population (CD44 ${ }^{+}$CD24) by 3-fold in MCF7 cells and 10-fold in T47D cells. Inhibition of endogenous miR-489 further increased the cancerous stem-like cell population by more than 3-fold in MCF7 cells and by more 
than 11-fold in T47D cells (Fig. 4E). Similarly, we observed increased MFE upon estrogen treatment and miR-489 inhibition not only increased MFE but also increased the mammosphere size. Forced expression of miR-489 almost completely prevented mammosphere formation (Fig. 4F). Together, these results suggest that estrogen regulated miR-489 is a feedback regulator that is able to confine estrogen-induced tumor cell growth and inhibit the population of cancerous stem-like cells.

\section{miR-489 inhibits estrogen induced signaling by targeting p38 and PTPN11}

We further seek to elucidate the molecular mechanism responsible for miR-489 mediated inhibition of estrogen-ERa axis. Multiple mechanisms have been identified for regulation of estrogen-ERa mediated gene expression $(24,25)$. Direct inhibition of ERa or its co-factors, inhibition of kinases that activate ERa, and inhibition of estrogen-induced nuclear localization of ERa have been previously reported to regulate estrogen induced gene transcription (26). We first examined if miR-489 exerted its effects by affecting localization of estrogen receptors. Interestingly, forced expression of miR-489 strongly promoted translocalization of estrogen receptors from the nucleus to the cytoplasm in MCF7 and T47D cell lines (Fig. 5A-B). In contrast, inhibition of endogenous miR-489 or knockout resulted in increased nuclear localization (additional file 2, Fig. S4A). Searching through factors which have been reported to regulate localization of estrogen receptors, we found that one of these factors, p38 MAPK, is a potential miR-489 target (26) (Fig. 5C). Interestingly, forced expression of miR-489 significantly downregulated total p38 MAPK (Fig. 5D). We then performed 3'UTR assay to examine if p38 MAPK is a direct target of miR-489. Forced expression of miR-489 significantly reduced the luciferase activity of wild type constructs but did not affect luciferase activity of constructs with a mutant miR-489 binding site (Fig. 5E). This result confirms that p38 MAPK is a direct target of miR-489. Next, we tested if the p38 MAPK inhibitor, SB203580, can phenocopy the effect of miR-489 on estrogen receptor localization. We transfected a control siRNA or miR-489 mimic for 72 hours or treated with DMSO or $10 \mu \mathrm{M} \mathrm{SB203580}$ for 24 hours in hormone starved cells followed by treatment with estrogen to examine estrogen-induced nuclear localization of estrogen receptors. Indeed, T47D cells treated with p38 MAPK inhibitor phenocopied the effect of miR-489 on ER localization (Additional file 2, Fig. S4B). However, co-treatment of MCF7 cells with a p38 inhibitor following miR-489 transfection could not further decrease ERa phosphorylation or enhance cytoplasmic translocation of ERa (Fig. 5F), indicating that miR-489 affects nuclear translocation of ERa at least partially by downregulating p38 MAPK.

Considering that phosphorylation of the ERa protein is also a critical step for its transcription activity, we investigated changes in the phosphorylation status upon modulation of miR-489 expression. Western blot analysis of total ERa protein and its phosphorylated forms suggest that forced expression of miR489 reduced the phosphorylation of ERa at both S118 and S167 sites (Fig. 5G). This data suggests that miR-489 may further regulate ligand-dependent activation of ERa through inhibition of kinases that phosphorylate ERa at residues S118 and S167. MAPK and AKT have been known to regulate ERa phosphorylation at S118 and S167 respectively. Indeed, miR-489 restoration significantly reduced the activated form of these kinases while its inhibition or knockout enhanced their activation (Fig. $5 \mathrm{G}$ ). 
All these three kinase pathways have been shown to be regulated by SHP2 and promote increase in tamoxifen resistance $(4,27)$. It is also known that the PTPN11 gene encoding SHP2 is the direct target of miR-489 $(16,28)$. To test whether miR-489 inhibits phosphorylation of all these kinases through regulating SHP2, we examined the effect of miR-489 modulation on SHP2 expression and the downstream signaling pathways in MCF7-HER2 and its isogenic cell line, MCF7-Vec, in the presence or absence of the SHP2 inhibitor, RMC4550 (Fig. 5H). Western blot analysis confirmed the upregulation of HER2, SHP2, pAKT and pERK in MCF7-HER2 cell line compared to MCF7-Vec. Transfection of miR-489 resulted in downregulation of SHP2 and p38 expression and decreased phosphorylation of AKT and MAPK. SHP2 inhibitor treatment alone inhibited phosphorylation of MAPK and AKT but had no effect on p38 expression or phosphorylation. SHP2 inhibitor treatment of miR-489-transfected cells showed some degree of synergy to inhibit phosphorylation of MAPK and AKT. Overall, our data suggested that miR-489 inhibits phosphorylation of MAPK and AKT at least partially though SHP2 but directly targets p38.

\section{Pharmacological inhibition of p38 MAPK, PI3K-Akt and MAPK phenocopies the effect of miR-489 in ER+ breast cancer cell lines}

Next, we examined whether pharmacological inhibition of all three responsible kinase signaling pathways phenocopies the effect of miR-489. We inspected estrogen-induced transcription and proliferation after inhibition of all three kinases. To a variable extent, we observed significant inhibition of transcriptional activity of ERa using an ERE-reporter cell line (Fig. 6A). qRT-PCR analysis also showed downregulation of estrogen responsive genes (Fig. 6B). Consistently, these data were also supported by diminished estrogen induced proliferation upon inhibition of all three kinases (Fig. 6C-6D). However, downregulation of endogenous miR-489 or miR-489 knockout was partially able to rescue cells from the growth inhibitory effect of kinase inhibitors (Fig. 6E-F). This data suggests that miR-489 may exert its inhibitory effect on estrogen signaling partially, if not completely, by simultaneously inhibiting p38 MAPK, AKT, and ERK signaling pathways.

Intriguingly, we observed that p38 MAPK inhibition had a significant inhibitory effect only in the presence of estrogen and did not affect estrogen-independent growth (Fig. 6G), indicating p38 MAPK activation may be estrogen dependent. Therefore, we tested if estrogen activates p38 MAPK and then mediates nuclear translocation. Since estrogen induced ERa nuclear translocation occurs within 5-30 minutes(29), we performed a time course of estrogen treatment on MCF7 and T47D cell lines. We observed a sharp increase in phospho-p38 MAPK and its downstream target, phosphor-ATF2, upon estrogen treatment in both cell lines (Fig. 6H). These results are consistent with previous studies that showed estrogen mediated activation of p38 MAPK in various tissues(26) (30). This provided evidence of a positive feedback loop between the E2-ERa axis and p38 MAPK in breast cancer cells such that binding of E2 leads to activation of p38 MAPK and activation of p38 MAPK leads to nuclear translocation of ERa which is necessary for its function as a transcription factor (Fig. 6l). As p38 MAPK activation was estrogen dependent, we therefore suspected p38 MAPK inhibition might have a pronounced effect in premenopausal women as compared to post-menopausal women (additional file 2, Fig. S5A). Indeed, we observed a higher p38 MAPK gene signature score in pre-menopausal luminal patients compared to post- 
menopausal luminal patients (Fig. 6J). Furthermore, luminal pre-menopausal patients with higher tumor grade also showed higher p38 MAPK gene signature (additional file 2, Fig. S5B). Additionally, our correlation analysis of miR-489 and p38 MAPK signature showed statistically significant inverse correlation in pre-menopausal patients. However, this correlation was reduced in post-menopausal patients (Fig. 6J). More importantly, high p38 MAPK expression in pre-menopausal patients predicted poor survival more significantly compared to post-menopausal patients (Fig. 6K). In summary, these results suggest that miR-489 regulates tamoxifen resistance by targeting multiple kinase signaling pathways and therefore could potentially be used as a therapeutic sensitizer to treat resistance patients.

\section{Discussion}

Defining the role of the differentially regulated miRNAs in breast cancer and drug-resistant cells could lead to the development of new diagnostic tools and therapeutic approaches. In the present study, we provided new evidence for the role of miR-489 in breast cancer and development of tamoxifen resistance. We demonstrated that expression of miR-489 is induced by estrogen and strongly correlates with cellular ERa status. The induction of miR-489 seems to mainly occur at the transcriptional level because the mRNA levels of its host gene, CALCR, are increased in several ER+ breast cancer cells upon estrogen treatment and correlate with miR-489 expression levels in breast cancers. However, the induction of CALCR gene expression appears more robust than the induction of miR-489 in the ER+ breast cancer cell lines MCF7 and T47D, indicating additional regulatory mechanisms may be involved. Apart from regulating the expression of miRNAs at the transcriptional level, there is evidence to suggest that ERa may be able to regulate microRNA processing and maturation. For example, ERa regulates the processing of the primary transcripts of the two miRNA clusters mir-17-92 and mir-106a-363, and therefore, miRNAs that are processed from the same precursor transcript accumulate in different relative amounts(31). Further studies are warranted to find out the detailed mechanisms of estrogen-regulated miR-489 expression in breast cancer cells.

Our previous studies have demonstrated that miR-489 functions as a tumor-suppressor microRNA in breast cancer $(13,16)$. Estrogen exposure is generally associated with increased risk for breast cancer. To better understand this apparent paradox, we further studied the signaling interplay between miR-489 and estrogen. Microarray analysis of the gene expression profiles of miR-489 overexpressing T47D cells indicated enriched ER-regulated signaling pathways. Consistently, in silico data analysis of gene expression in large cohorts of breast cancer samples confirmed the inverse correlation between miR-489 signature and estrogen responsive genes. Molecular experiments validated that overexpression of miR489 suppressed ERa signaling pathways. These results led us to hypothesize that induction of miR-489 by estrogen may serve as a fail-safe mechanism to prevent over activation of ERa downstream signaling pathways in response to high levels of estrogen. The corollary of this hypothesis is that the loss of miR489 will promote estrogen-induced tumorigenesis or aggressiveness in cancer cells. Indeed, we observed that the inhibition of endogenous miR-489 robustly enhanced estrogen-induced cell proliferation and expansion of cancer stem-like cell populations, which is in contrast to the relatively mild effects of overexpression of miR-489 in breast cancer cells.

Page $11 / 26$ 
Previous studies have described a negative feedback loop between ERa and several miRNAs that are induced upon estrogenic stimulation and that downregulate ERa (31,32). For example, estrogen-induced expression of the miR-1792 family of microRNAs, which can restrict estrogen actions in feedback through different ways. While several microRNAs like miR-18a, miR-19b, and miR-20b can directly downregulate ERa expression and other microRNAs such as miR-20a, miR-17-5p, miR-106a, and miR-20b downregulate expression of ERa transcriptional co-factor AIB1(31). ERa is not a predicted target of miR-489. We did not observe any changes in its expression levels after manipulations of miR489 as expected. However, we found that overexpression of miR-489 drastically suppressed ERa phosphorylation at ser118 and Ser167. Given that AKT and ERK kinases are known to phosphorylate ERa at Ser118 and Ser167 respectively and enhances its transcriptional actively (9,33-37), one mechanism of miR-489 mediated restriction of estrogen activities is through the inhibition of MAPK and AKT activities. In addition, previous studies have demonstrated that p38 kinase can phosphorylate ERa and affect its cellular localization(18,30,38). p38 is one of the predicted target genes of miR-489, which was validated by transient transfection of a p38 promoter reporter assay. Consistently, overexpression of miR-489 resulted in translocation of ERa from nucleus to cytoplasm, adding another mechanism of miR-489 restriction of estrogen action.

Interestingly, we also observed a positive feedback loop between E2-ERa signaling and p38 MAPK. Estrogen activates p38 MAPK within a few minutes upon binding to its receptor ERa.This activated p38 MAPK leads to nuclear translocation of Era, which is essential for its transcriptional activity. Similar observations have also been reported for ERK and AKT in regard to estrogen signaling $(39,40)$. Like p38 MAPK, estrogen also activates AKT and ERK(41). Activated ERK and AKT then activate ERa by phosphorylating ERa at Ser1018 and Ser167, respectively (42). miR-489 regulates this positive feedback loop by inhibiting p38 MAPK, AKT, and ERK activity.

We posit that the physical function of the negative feedback loop between ERa and the miR-489 would be to create a fail-safe mechanism to avoid high ERa activity. High ERa activity is potentially dangerous for the cell and is a major risk factor for breast cancer. In addition, miR-489 could serve as a potential prognostic marker in ER+ breast cancer where ER+ patients with low miR-489 may possess hyper activation of E2-ERa signaling and may potentially represent aggressive cancers. Indeed, clinical analysis of ER+ breast cancer patients suggest that patients with low miR-489 expression have worse survival rates and are more likely to develop drug resistance. Despite a strong correlation between expression of ERa and a favorable response to endocrine therapy, 40-50\% of patients with ERa+ breast cancer develop resistance or exhibit de novo resistance, and patients with luminal B and ERa+/PGR- breast cancer exhibit a poor response to tamoxifen(43). The underlying mechanism appears to be deregulation in estrogen receptor signaling pathways due to crosstalk of growth factor signaling pathways such as $\mathrm{PI} 3 \mathrm{~K} / \mathrm{AKT} / \mathrm{mTOR}$ and epidermal growth factor receptor (EGFR) crosstalk with ERa signaling to enhance pro-proliferative ERa regulated gene expression and suppression of PGR gene expression(9,18,44-46). Our observations here indicate that miR-489 could potentially serve as a useful therapy sensitizer to treat tamoxifen resistance tumors. First, our study along with other reports showed miR-489 is significantly downregulated in tamoxifen-resistant cell lines. Intriguingly, it lost both acquired and de novo resistance. Second, miR-489 directly inhibits AKT and MAPK pathways, which are well established mechanisms that 
promote estrogen independent growth and tamoxifen resistance. Third, miR-489 directly targets HER2 and its downstream molecules including SHP2 and AKT(16). HER2 overexpression is known to confer tamoxifen resistance through increased bidirectional ER/HER2 cross-talk $(4,18,47,48)$. Fourth, p38 MAPK can potentiate the ER in part through increased phosphorylation of ER at Thr311 (26), and enhance ER signaling through coactivator regulation(38). Increased p38 activity has been associated with breast cancer drug resistance and invasion(18). Our results suggest that miR-489 directly targets $p 38$ to break the positive feedback loop between ER and p38. Furthermore, autophagy and EMT have been reported to promote tamoxifen resistance. MCF7-TAM cells have been reported to undergo EMT and possesses higher basal autophagy $(10,49)$. Inhibition of both processes have been previously shown to reverse the tamoxifen resistance. Interestingly, miR-489 has previously been reported to reverse EMT through inhibition of Smad3 expression which leads to sensitization of doxorubicin(50). Previously, we have reported its role in autophagy and chemo-resistance(13). This data supports a general role of miR-489 in the modulation of drug resistance.

\section{Conclusions}

In summary, this study revealed a new negative feedback loop between miR-489 and estrogen and demonstrated the potential role of miR-489 in the development of ER+ breast cancer and tamoxifen resistance. miR-489-based therapy may be a useful adjuvant therapy not only in tamoxifen-resistant patients but also in treatment naïve patients since apart from inhibiting estrogen signaling, it also blocks HER2-PI3K-AKT and MAPK pathways and may potentially reduce emergence of resistance to tamoxifen. These results contribute to the understanding of the complex regulatory pathways regulating ERa activity and may provide insights needed to develop novel ER+ breast cancer therapies.

\section{Abbreviations}

miR-489: microRNA 489

miRNA: microRNA

ER+: Estrogen receptor positive

ERa: Estrogen receptor a

ESR1: Estrogen Receptor 1

EGFR: Epidermal growth factor receptor

HER2: Human epidermal growth factor receptor 2

LBD: Ligand-binding domain

qRT-PCR: Quantitative reverse transcription polymerase chain reaction 
siRNA: Small interfering RNA

GFP: Green fluorescent protein

CALCR: Calcitonin Receptor

PGR: Progesterone Receptor

ERBB: Epidermal growth factor

TFF1: Trefoil factor 1

CXCL12: C-X-C motif chemokine ligand 12

TAM: Tamoxifen

MFE: Mammosphere forming efficiency

E2: Estradiol

KO: Knockout

CRISPR: Clustered regularly interspaced short palindromic repeats

Cas9: CRISPR-associated protein 9

PBS: Phosphate-buffered saline

MAPK: Mitogen-activated protein kinase

AKT: Protein kinase B

PI3K: Phosphoinositide 3-kinase

SHP2: Protein tyrosine phosphatase

mTOR: Mammalian target of rapamycin

ERK: Extracellular signal-regulated kinase

\section{Declarations}

\section{Ethical Approval and Consent to participate}

Not applicable

Consent for publication 
Not applicable

\section{Availability of supporting data}

The microarray data in this manuscript is available on the GEO database (GSE99728)..

\section{CONFLICT OF INTEREST}

The authors declare no conflict of interest.

\section{Funding}

This work was partially supported by the NIH grants (R01CA178386, R21CA252360) and the USC ASPIRE1 grant to HC, R01CA218578 to DF, NSF grant (No.1853365) to XL and HC, the American Cancer Society Research Scholar Grant RSG-19-194-01-CSM to OS, the USC ASPIRE post-doctoral fellowship to SL and the USC SPARC graduate fellowship to MS.

\section{ACKNOWLEDGEMENTS}

We thank Dr. Saraswati Sukumar (John Hopkins University School of Medicine) for providing the breast cancer cell lines, Dr. Rachel Schiff (Baylor College of Medicine) for providing the MCF7-Vector and MCF7HER2 cell lines, Dr. Philip Buckhaults (University of South Carolina) for providing the Cas9-GFP plasmid.

\section{Author information}

\section{Affiliations}

Department of Biological Science, Center for Colon Cancer Research, University of South Carolina, Columbia, SC 29208.

Mihtil Soni, Gourab Gupta, Yogin Patel, Manikanda Raja Keerthi Raja \& Hexin Chen

Department of Drug Discovery and Biomedical Sciences, South Carolina College of Pharmacy, University of South Carolina, Columbia, SC 29208.

Ozge Saatci, Peisheng Xu \& Ozgur Sahin

Department of Chemistry and Biocehmistry University of South Carolina, Columbia, SC29201.

Jie Li

Department of Mathematics, University of South Carolina, Columbia, SC29201

Xinfeng Liu

Department of Biomedical Engineering, Stevens Institute of Technology, Hoboken, NJ, 07030. 
Hongjun Wang

Department of Cell Biology and Anatomy, University of South Carolina School of Medicine, Columbia, SC 29209.

Daping Fan

\section{Corresponding authors}

Correspondence to Hexin Chen.

\section{Authors' contributions}

\section{Contributions}

M.S. designed experiments, conducted experiments, analyzed data, and drafted the manuscript. M.S., O.S.,G.G., Y.P. and M.K. conducted experiments and analyzed data. X.L., J.L., P.X., H.W., D.F. and O.S. interpreted data and edited manuscript. H.C. and M.S. designed experiments, analyzed data, and wrote the manuscript. The authors read and approved the final manuscript.

\section{References}

1. Nass N, Kalinski T. Tamoxifen resistance: from cell culture experiments towards novel biomarkers. Pathology, research and practice 2015;211:189-97

2. Clarke R, Tyson JJ, Dixon JM. Endocrine resistance in breast cancer--An overview and update. Molecular and cellular endocrinology 2015;418 Pt 3:220-34

3. Jeselsohn R, Buchwalter G, De Angelis C, Brown M, Schiff R. ESR1 mutations-a mechanism for acquired endocrine resistance in breast cancer. Nature reviews Clinical oncology 2015;12:573-83

4. Osborne CK, Schiff R. Mechanisms of endocrine resistance in breast cancer. Annu Rev Med 2011;62:233-47

5. Fan P, Maximov PY, Curpan RF, Abderrahman B, Jordan VC. The molecular, cellular and clinical consequences of targeting the estrogen receptor following estrogen deprivation therapy. Molecular and cellular endocrinology 2015;418 Pt 3:245-63

6. Rugo HS, Rumble RB, Macrae E, Barton DL, Connolly HK, Dickler MN, et al. Endocrine Therapy for Hormone Receptor-Positive Metastatic Breast Cancer: American Society of Clinical Oncology Guideline. Journal of clinical oncology : official journal of the American Society of Clinical Oncology 2016;34:3069-103

7. Finn RS, Aleshin A, Slamon DJ. Targeting the cyclin-dependent kinases (CDK) 4/6 in estrogen receptor-positive breast cancers. Breast cancer research : BCR 2016;18:17

8. Reinert T, Saad ED, Barrios CH, Bines J. Clinical Implications of ESR1 Mutations in Hormone Receptor-Positive Advanced Breast Cancer. Frontiers in oncology 2017;7:26 
9. Shou J, Massarweh S, Osborne CK, Wakeling AE, Ali S, Weiss H, et al. Mechanisms of tamoxifen resistance: increased estrogen receptor-HER2/neu cross-talk in ER/HER2-positive breast cancer. J Natl Cancer Inst 2004;96:926-35

10. Ward A, Balwierz A, Zhang JD, Kublbeck M, Pawitan Y, Hielscher T, et al. Re-expression of microRNA375 reverses both tamoxifen resistance and accompanying EMT-like properties in breast cancer. Oncogene 2013;32:1173-82

11. Bailey ST, Westerling T, Brown M. Loss of estrogen-regulated microRNA expression increases HER2 signaling and is prognostic of poor outcome in luminal breast cancer. Cancer Res 2015;75:436-45

12. Raza U, Saatci O, Uhlmann S, Ansari SA, Eyupoglu E, Yurdusev E, et al. The miR-644a/CTBP1/p53 axis suppresses drug resistance by simultaneous inhibition of cell survival and epithelialmesenchymal transition in breast cancer. Oncotarget 2016;7:49859-77

13. Soni M, Patel Y, Markoutsa E, Jie C, Liu S, Xu P, et al. Autophagy, Cell Viability, and Chemoresistance Are Regulated By miR-489 in Breast Cancer. Mol Cancer Res 2018;16:1348-60

14. Patel Y, Soni M, Awgulewitsch A, Kern MJ, Liu S, Shah N, et al. Overexpression of miR-489 derails mammary hierarchy structure and inhibits HER2/neu-induced tumorigenesis. Oncogene 2019;38:44553

15. Liu S, Lee JS, Jie C, Park MH, Iwakura Y, Patel Y, et al. HER2 Overexpression Triggers an IL1alpha Proinflammatory Circuit to Drive Tumorigenesis and Promote Chemotherapy Resistance. Cancer Res 2018;78:2040-51

16. Patel Y, Shah N, Lee JS, Markoutsa E, Jie C, Liu S, et al. A novel double-negative feedback loop between miR-489 and the HER2-SHP2-MAPK signaling axis regulates breast cancer cell proliferation and tumor growth. Oncotarget 2016;7:18295-308

17. Loi S, Haibe-Kains B, Desmedt C, Lallemand F, Tutt AM, Gillet C, et al. Definition of clinically distinct molecular subtypes in estrogen receptor-positive breast carcinomas through genomic grade. Journal of clinical oncology : official journal of the American Society of Clinical Oncology 2007;25:1239-46

18. Gutierrez MC, Detre S, Johnston S, Mohsin SK, Shou J, Allred DC, et al. Molecular changes in tamoxifen-resistant breast cancer: relationship between estrogen receptor, HER-2, and p38 mitogenactivated protein kinase. Journal of clinical oncology : official journal of the American Society of Clinical Oncology 2005;23:2469-76

19. Davies MP, Barraclough DL, Stewart C, Joyce KA, Eccles RM, Barraclough R, et al. Expression and splicing of the unfolded protein response gene XBP-1 are significantly associated with clinical outcome of endocrine-treated breast cancer. International journal of cancer 2008;123:85-8

20. Cook KL, Shajahan AN, Clarke R. Autophagy and endocrine resistance in breast cancer. Expert Rev Anticancer Ther 2011;11:1283-94

21. Clarke R, Cook KL. Unfolding the Role of Stress Response Signaling in Endocrine Resistant Breast Cancers. Front Oncol 2015;5:140

22. Fillmore CM, Gupta PB, Rudnick JA, Caballero S, Keller PJ, Lander ES, et al. Estrogen expands breast cancer stem-like cells through paracrine FGF/Tbx3 signaling. Proc Natl Acad Sci U S A 
2010;107:21737-42

23. Clarke MF, Fuller M. Stem cells and cancer: two faces of eve. Cell 2006;124:1111-5

24. Carroll JS, Brown M. Estrogen receptor target gene: an evolving concept. Molecular endocrinology (Baltimore, Md) 2006;20:1707-14

25. Bjornstrom L, Sjoberg M. Mechanisms of estrogen receptor signaling: convergence of genomic and nongenomic actions on target genes. Molecular endocrinology (Baltimore, Md) 2005;19:833-42

26. Lee $\mathrm{H}$, Bai W. Regulation of estrogen receptor nuclear export by ligand-induced and p38-mediated receptor phosphorylation. Mol Cell Biol 2002;22:5835-45

27. Zhang J, Zhang F, Niu R. Functions of Shp2 in cancer. J Cell Mol Med 2015;19:2075-83

28. Kikkawa N, Hanazawa T, Fujimura L, Nohata N, Suzuki H, Chazono H, et al. miR-489 is a tumoursuppressive miRNA target PTPN11 in hypopharyngeal squamous cell carcinoma (HSCC). Br J Cancer 2010;103:877-84

29. Song RX, Barnes CJ, Zhang Z, Bao Y, Kumar R, Santen RJ. The role of Shc and insulin-like growth factor 1 receptor in mediating the translocation of estrogen receptor alpha to the plasma membrane. Proceedings of the National Academy of Sciences of the United States of America 2004;101:2076-81

30. Seval Y, Cakmak H, Kayisli UA, Arici A. Estrogen-mediated regulation of p38 mitogen-activated protein kinase in human endometrium. J Clin Endocrinol Metab 2006;91:2349-57

31. Castellano L, Giamas G, Jacob J, Coombes RC, Lucchesi W, Thiruchelvam P, et al. The estrogen receptor-alpha-induced microRNA signature regulates itself and its transcriptional response. Proc Natl Acad Sci U S A 2009;106:15732-7

32. Pandey DP, Picard D. Multidirectional interplay between nuclear receptors and microRNAs. Curr Opin Pharmacol 2010;10:637-42

33. Oh AS, Lorant LA, Holloway JN, Miller DL, Kern FG, El-Ashry D. Hyperactivation of MAPK induces loss of ERalpha expression in breast cancer cells. Molecular endocrinology (Baltimore, Md) 2001;15:134459

34. Yue W, Fan P, Wang J, Li Y, Santen RJ. Mechanisms of acquired resistance to endocrine therapy in hormone-dependent breast cancer cells. J Steroid Biochem Mol Biol 2007;106:102-10

35. Wong CW, McNally C, Nickbarg E, Komm BS, Cheskis BJ. Estrogen receptor-interacting protein that modulates its nongenomic activity-crosstalk with Src/Erk phosphorylation cascade. Proceedings of the National Academy of Sciences of the United States of America 2002;99:14783-8

36. Miller PC, Clarke J, Koru-Sengul T, Brinkman J, El-Ashry D. A novel MAPK-microRNA signature is predictive of hormone-therapy resistance and poor outcome in ER-positive breast cancer. Clinical cancer research : an official journal of the American Association for Cancer Research 2015;21:373-85

37. Thottassery JV, Sun Y, Westbrook L, Rentz SS, Manuvakhova M, Qu Z, et al. Prolonged extracellular signal-regulated kinase $1 / 2$ activation during fibroblast growth factor 1 - or heregulin beta1-induced antiestrogen-resistant growth of breast cancer cells is resistant to mitogen-activated protein/extracellular regulated kinase kinase inhibitors. Cancer Res 2004;64:4637-47 
38. Frigo DE, Basu A, Nierth-Simpson EN, Weldon CB, Dugan CM, Elliott S, et al. p38 mitogen-activated protein kinase stimulates estrogen-mediated transcription and proliferation through the phosphorylation and potentiation of the p160 coactivator glucocorticoid receptor-interacting protein 1. Molecular endocrinology (Baltimore, Md) 2006;20:971-83

39. Bunone G, Briand PA, Miksicek RJ, Picard D. Activation of the unliganded estrogen receptor by EGF involves the MAP kinase pathway and direct phosphorylation. EMBO J 1996;15:2174-83

40. Anbalagan M, Rowan BG. Estrogen receptor alpha phosphorylation and its functional impact in human breast cancer. Mol Cell Endocrinol 2015;418 Pt 3:264-72

41. Filardo EJ, Quinn JA, Bland KI, A. Raymond Frackelton J. Estrogen-Induced Activation of Erk-1 and Erk-2 Requires the G Protein-Coupled Receptor Homolog, GPR30, and Occurs via Trans-Activation of the Epidermal Growth Factor Receptor through Release of HB-EGF. Molecular Endocrinology 2000;14:1649-60

42. Lannigan DA. Estrogen receptor phosphorylation. Steroids 2003;68:1-9

43. Cui X, Schiff R, Arpino G, Osborne CK, Lee AV. Biology of progesterone receptor loss in breast cancer and its implications for endocrine therapy. Journal of clinical oncology : official journal of the American Society of Clinical Oncology 2005;23:7721-35

44. Jin K, Kong X, Shah T, Penet MF, Wildes F, Sgroi DC, et al. The HOXB7 protein renders breast cancer cells resistant to tamoxifen through activation of the EGFR pathway. Proc Natl Acad Sci U S A 2012;109:2736-41

45. Santen RJ, Fan P, Zhang Z, Bao Y, Song RX, Yue W. Estrogen signals via an extra-nuclear pathway involving IGF-1R and EGFR in tamoxifen-sensitive and -resistant breast cancer cells. Steroids 2008

46. Aesoy R, Sanchez BC, Norum JH, Lewensohn R, Viktorsson K, Linderholm B. An autocrine VEGF/VEGFR2 and p38 signaling loop confers resistance to 4-hydroxytamoxifen in MCF-7 breast cancer cells. Mol Cancer Res 2008;6:1630-8

47. Yang Z, Barnes CJ, Kumar R. Human Epidermal Growth Factor Receptor 2 Status Modulates Subcellular Localization of and Interaction with Estrogen Receptor a in Breast Cancer Cells. Clinical Cancer Research 2004;10:3621-8

48. Montgomery RB, Makary E, Schiffman K, Goodell V, Disis ML. Endogenous anti-HER2 antibodies block HER2 phosphorylation and signaling through extracellular signal-regulated kinase. Cancer Res 2005;65:650-6

49. Nagelkerke A, Sieuwerts AM, Bussink J, Sweep FC, Look MP, Foekens JA, et al. LAMP3 is involved in tamoxifen resistance in breast cancer cells through the modulation of autophagy. Endocr Relat Cancer 2014;21:101-12

50. Jiang L, He D, Yang D, Chen Z, Pan Q, Mao A, et al. MiR-489 regulates chemoresistance in breast cancer via epithelial mesenchymal transition pathway. FEBS Lett 2014;588:2009-15

\section{Figures}


A.

B.
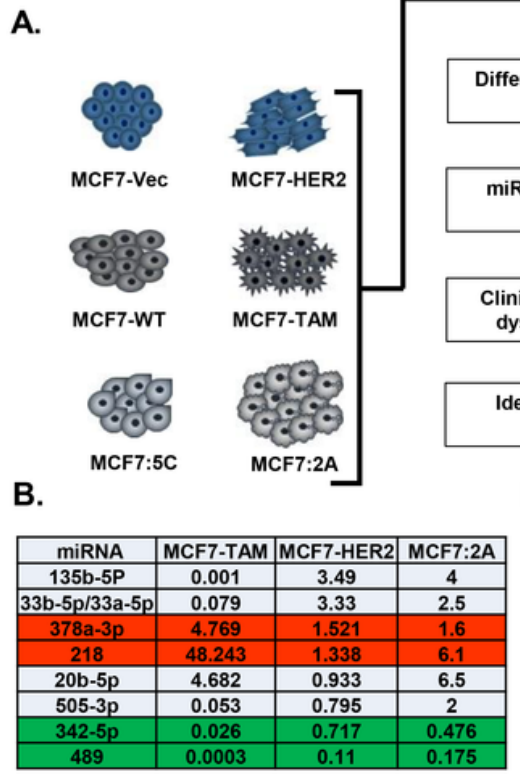

c.

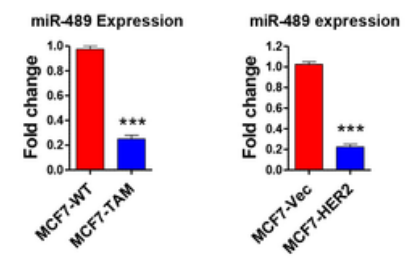

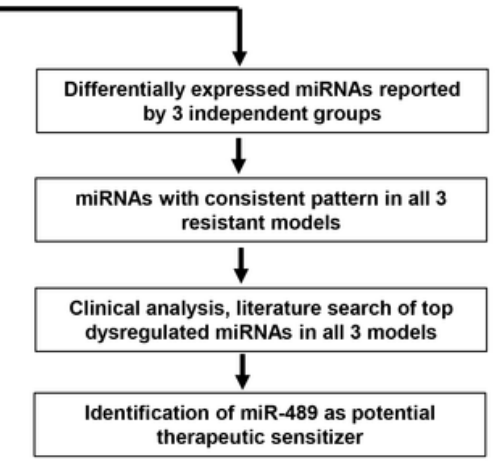

D.
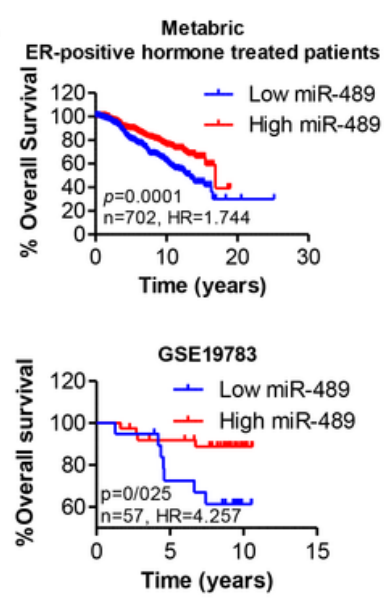

E.

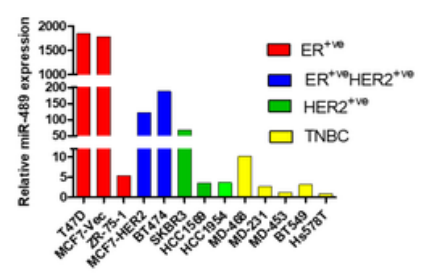

G.
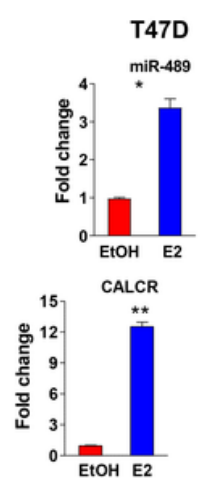

H.

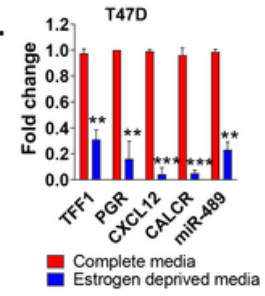

F.

\begin{tabular}{|c|c|c|}
\hline $\begin{array}{c}\text { Gene } \\
\text { Identifier }\end{array}$ & $\begin{array}{c}\text { Correlation } \\
\text { coefficient }\end{array}$ & $\begin{array}{c}\text { Parametric } \\
\text { p-value }\end{array}$ \\
\hline CALCR & 0.558 & 0 \\
\hline ESR1 & 0.208 & 0 \\
\hline PGR & 0.268 & 0 \\
\hline
\end{tabular}

\begin{tabular}{l|l} 
PGR & 0.268
\end{tabular}
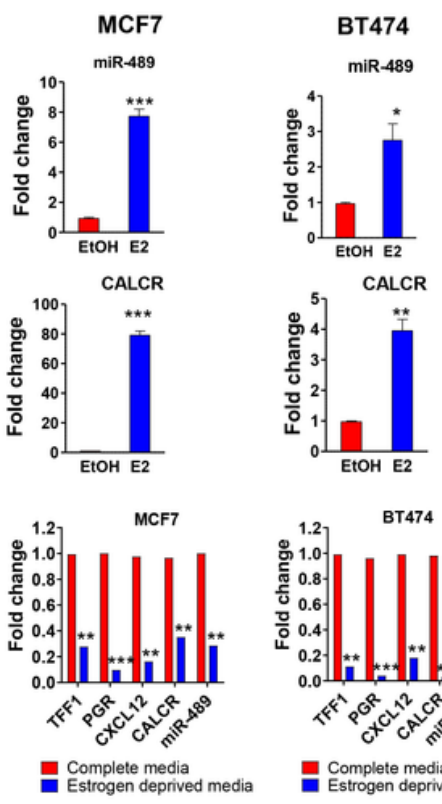

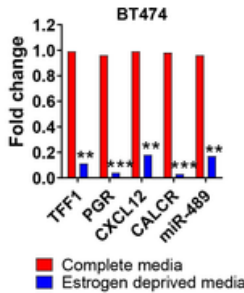

Figure 1

Figure 1

miR-489 expression is lost in tamoxifen resistance, predicts breast cancer aggressiveness and is regulated by estrogen/ERa axis. A. Schematic diagram for the identification and validation of miRNAs involved in tamoxifen resistance. miRNA analysis from three independent studies using three independent tamoxifen resistant model systems. B. List of top dysregulated miRNAs in all three Tamoxifen resistant cell lines. C. qRT-PCR validation of miR-489 in MCF7-TAM and MCF7-HER2 cell line. D. Clinical analysis of two datasets analyzing miR-489 expression in ER-positive breast cancer patients receiving hormone therapy. E. miR-489 expression in breast cancer cell lines. F. Correlation of miR-489 expression with expression of ERa and PGR. G. qRT-PCR analysis of miR-489 expression upon estrogen stimulation in three ER+ breast cancer cell lines. H. qRT-PCR analysis of miR-489 expression upon estrogen deprivation in three ER+ breast cancer cell lines. ${ }^{\star *}, p<0.01 ; * \star *, p<0.001$. Data are representative of three independent experiments. 


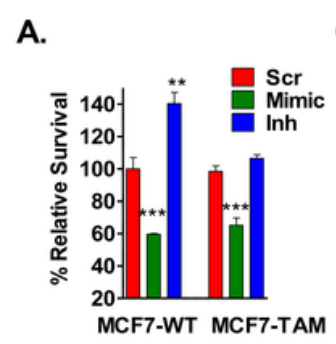

B.

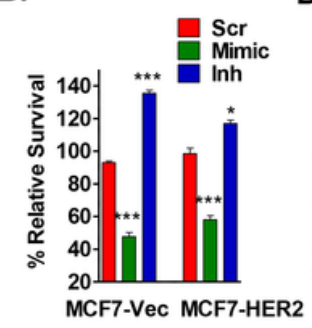

C.
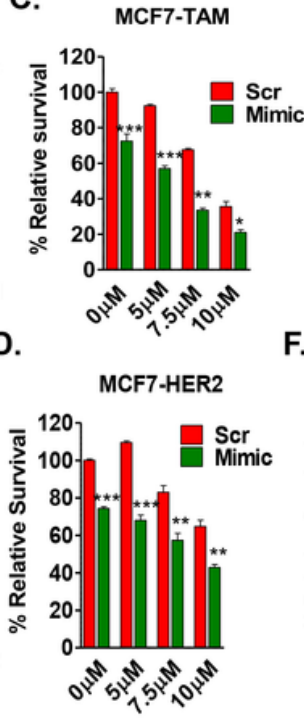

E.
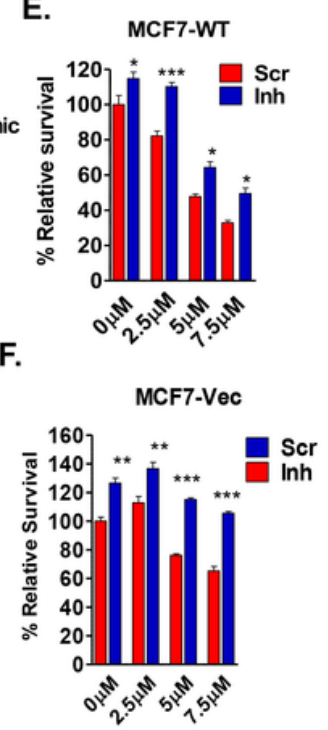

G.
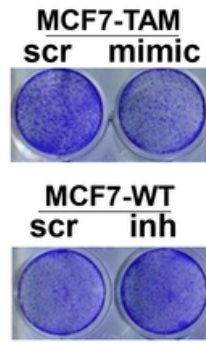

H.

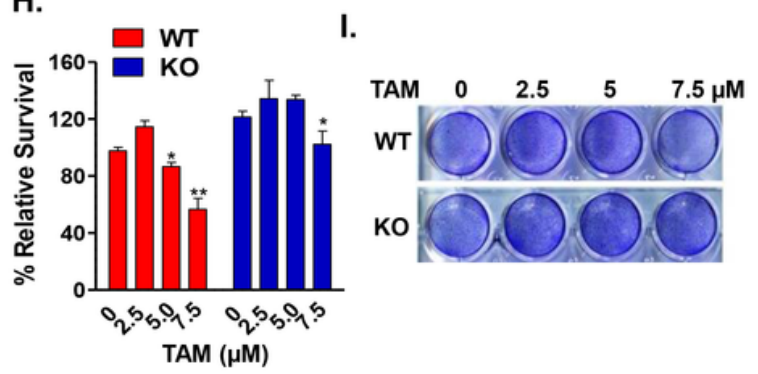

Figure 2

Figure 2

miR-489 restoration overcomes acquired and de novo tamoxifen resistance.

A-B. Effect of miR-489 modulation on proliferation of two pairs of tamoxifen resistant cell lines. Cells were transfected with 28nM of Scramble siRNA, miR-489 mimic and miR-489 inhibitor for 72hours followed by MTT based viability assay. C-D. miR-489 restoration sensitizes MCF7-TAM and MCF7-HER2 cell lines to tamoxifen. Scramble siRNA or miR-489 mimic was transfected with or without Tamoxifen for 72hours followed by MTT based viability assay. E-F Depletion of miR-489 promotes tamoxifen resistance in tamoxifen sensitive cell line MCF7-TAM and MCF7-HER2 cell lines to tamoxifen. G. Colony formation assay showing miR-489 modulates tamoxifen resistance. Cells were treated with indicated microRNA mimics or inhibitors with or without Tamoxifen for 72 hours followed by colony formation assay for 7-10 days. H-I. miR-489 knockout confers tamoxifen resistance. WT and KO T47D cells were treated with indicated concentration of tamoxifen and viability was examined using MTT assay $(\mathbf{H})$ and colony formation assay $(\mathrm{I}) .{ }^{*}, \mathrm{p}<0.01 ; * \star \star, p<0.001$. Data are representative of three independent experiments. 
A.

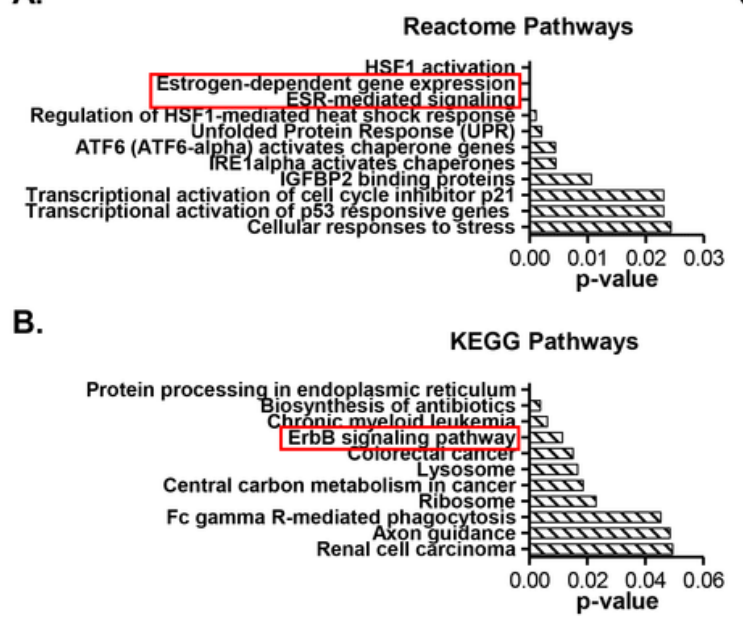

C.

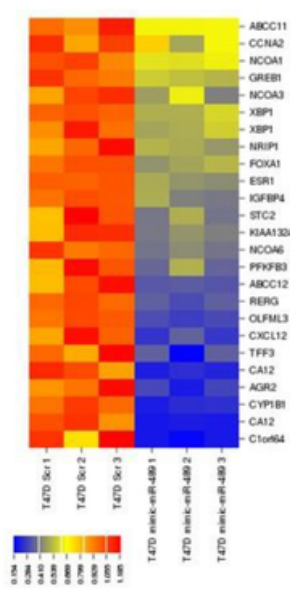

D.

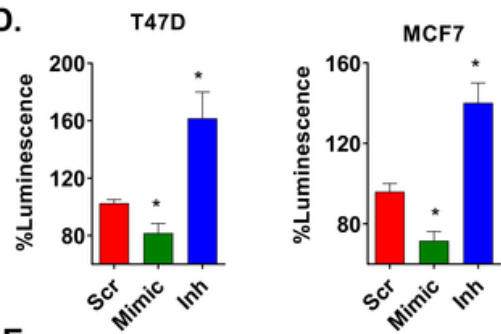

E.

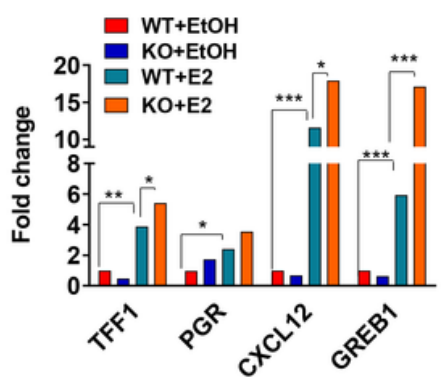

F.

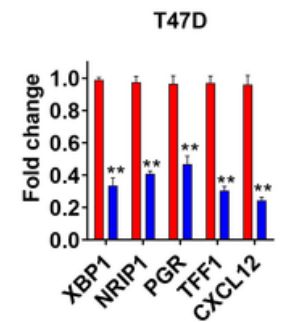

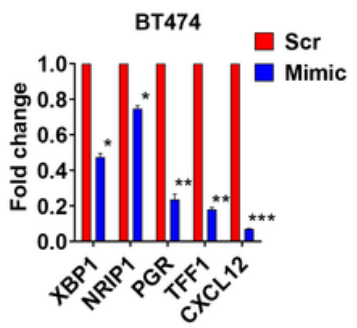
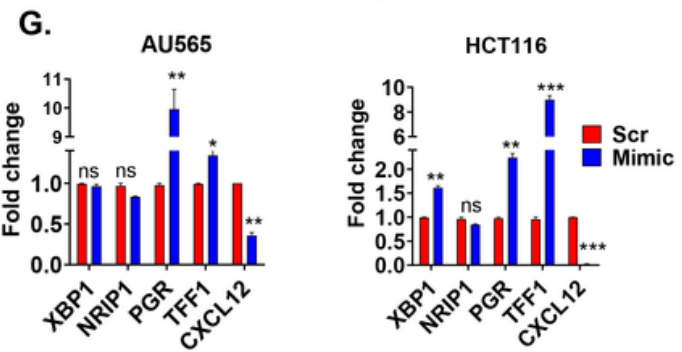

Figure 3

\section{Figure 3}

Gene Expression Analysis revealed enrichment of multiple pathways involved in estrogen signaling and tamoxifen resistance

A-B. Whole transcriptome analysis of miR-489 transfected and control T47D cells followed by pathway enrichment analysis revealed multiple pathways involved in tamoxifen resistance including ESRmediated signaling and ErBB2 pathways. C. Heatmap demonstrating downregulation of multiple estrogen responsive genes in miR-489 transfected T47D cells. D. miR-489 negatively regulates estrogen induced transcription by transient transfection of cells with ERE-reporter system. E. miR-489 WT and knockout T47D cells were treated with ethanol or estrogen for 6 hours and expression of estrogen responsive genes was examined using qRT-PCR. F-G. qRT-PCR analysis of estrogen responsive genes upon miR-489 restoration shows downregulation of estrogen responsive genes only in ER+ breast cancer cell lines. ${ }^{*}, p<0.05 ; * \star, p<0.01 ; * \star \star, p<0.001$. Data are representative of three independent experiments. 
A.

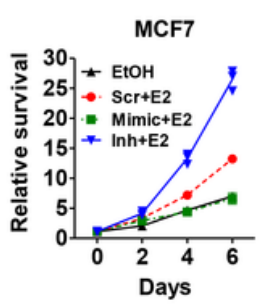

E.

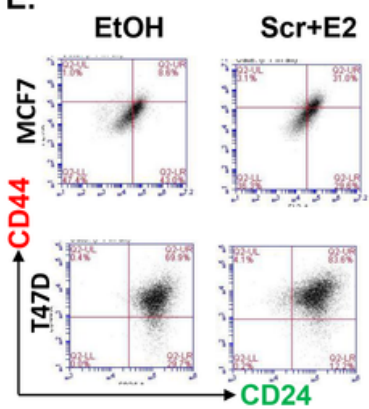

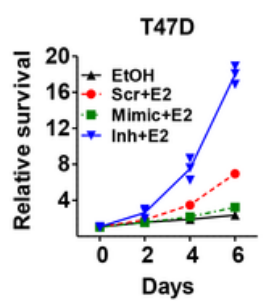

Mimic+E2 Inh+E2
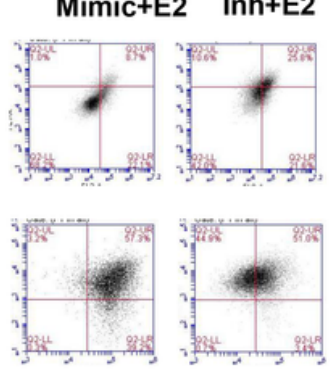

B.
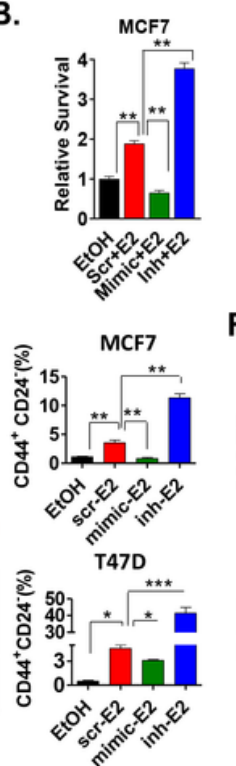

C.

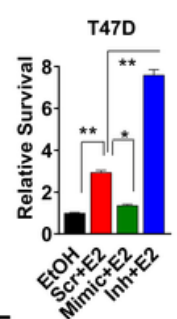

F.
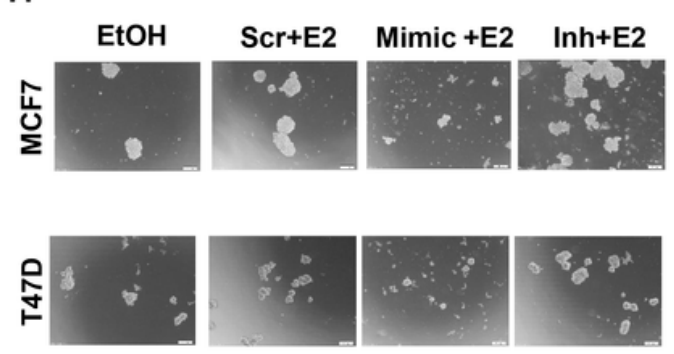

E2

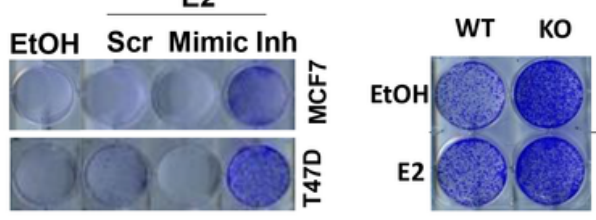

MCF7

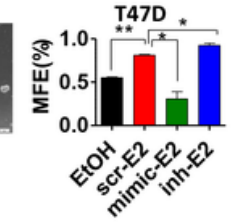

Figure 4

\section{Figure 4}

miR-489 acts as an endogenous negative feedback loop to dampen estrogen activities. A. miR-489 restoration inhibits proliferation of ER+ breast cancer cell line while inhibition of endogenous miR-489 dramatically enhances proliferation of MCF7 and T47D. Scramble siRNA, miR-489 mimic or miR-489 inhibitor was transfected in presence or absence of estrogen for 72 hours followed by MTT based viability assay. B. Quantification of viable cells on day 6 post treatment of MCF7 and T47D. C. Inhibition of endogenous miR-489 dramatically enhances estrogen induced colony formation. MCF7 and T47D cells were transfected with scramble siRNA, miR-489 mimic and miR-489 inhibitor in presence or absence of estrogen for followed by colony formation assay. D. Hormone starved miR-489 WT and KO T47D cells were seeded in 12- well plate and treated with ethanol or E2 for 6-days and cell viability was measured by crystal violet staining. E. Breast cancer cell lines were transfected with scr, mimic or inhibitor in presence of estrogen for $72 \mathrm{hrs}$ followed by flow cytometry to examine CD24 and CD 44 surface markers. F. Breast cell lines were transfected with scr, mimic or inhibitor in presence of estrogen for $72 \mathrm{hrs}$ followed by mammosphere assay. Scr. Scramble control; Inh. Inhibitor of miR-489. **, $p<0.01 ; \star \star \star, p<0.001$. Data are representative of three independent experiments. 
A.

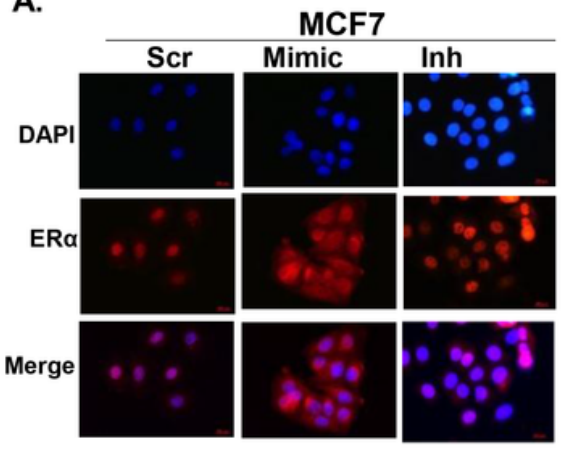

B.

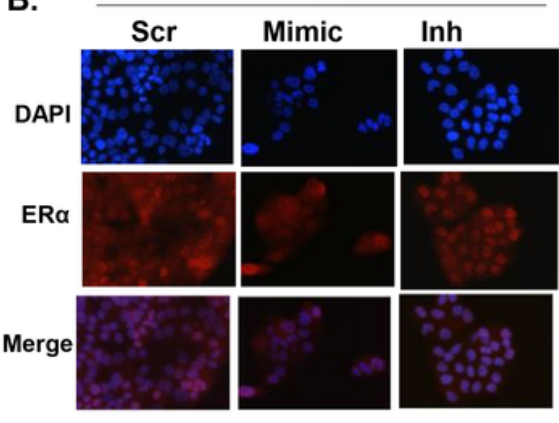

C.

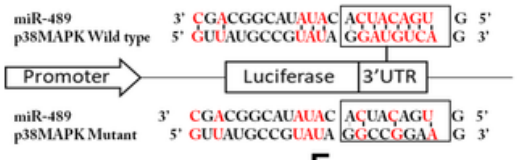

D.

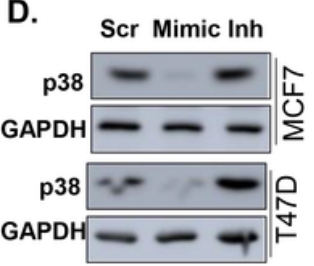

E.

G.
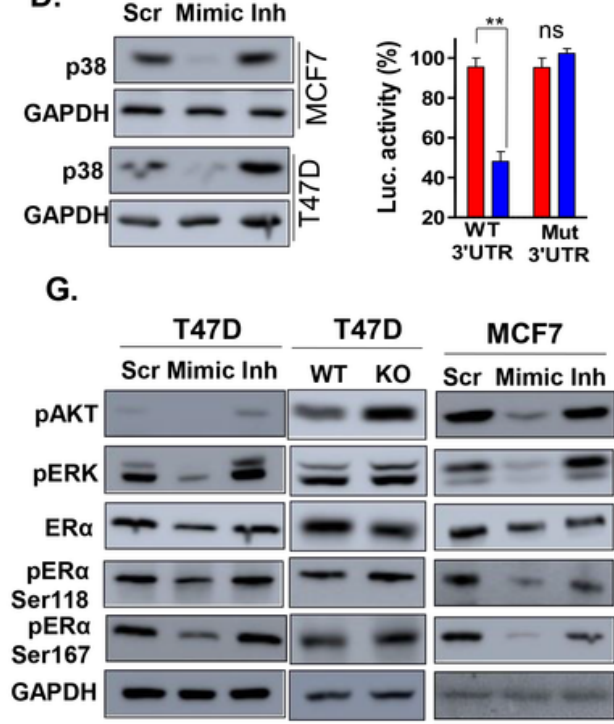

F.

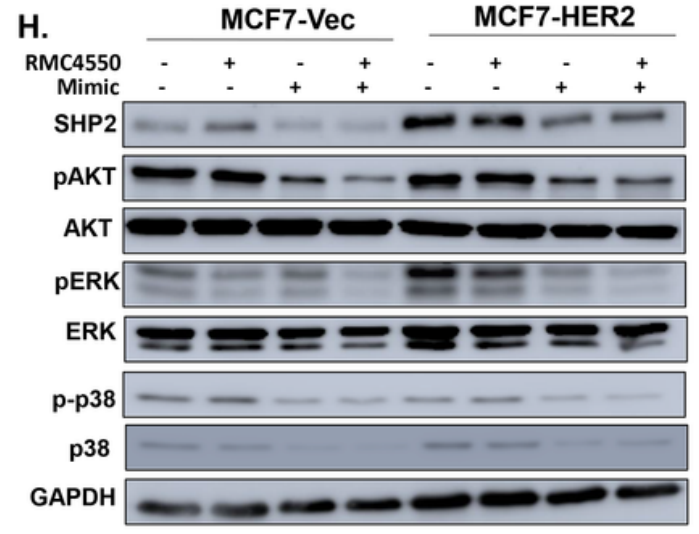

Figure 5

\section{Figure 5}

miR-489 inhibits estrogen induced signaling by inhibiting p38 MAPK, PI3K-AKT and MAPK-ERK pathways. A-B. MCF7 and T47D cells were transfected with scramble, mimic or inhibitor for $72 \mathrm{hrs}$ in estrogen deprived media followed by estrogen stimulation for 15 minutes. Data represents localization of estrogen receptor using immunofluorescence. C. miR-489 binding site in p38 MAPK 3' UTR. D. scramble, mimic or inhibitor was transfected, and western blot analysis was performed to examine total p38 MAPK protein. E. 3' UTR transfection assay showing miR-489 directly binds to 3'UTR of p38 MAPK. Data are means of three replicates \pm SEM. F. MCF7 cells were transfected with scramble or mimic in the presence of DMSO or p38 MAPK inhibitor SB23508. At 72 hrs post transfection, cytoplasmic and nuclear fractionations were prepared for western blot analysis of expression and phosphorylation statuses of ERa and p38. G. Cells were transfected with scramble, mimic or inhibitor for 72 hours and ERa phosphorylation and responsible kinases were examined using western blot analysis. H. MCF7 cells were transfected with scramble or mimic in the presence of DMSO or SHP2 allosteric inhibitor RMC4550. ERa phosphorylation and responsible kinases were examined using western blot analysis. 
A.

B.

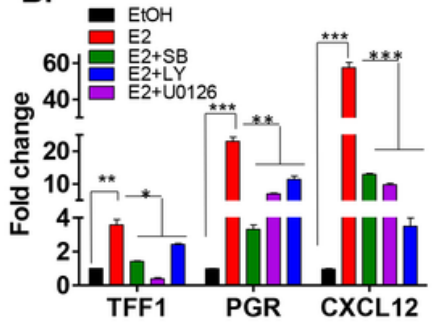

C.

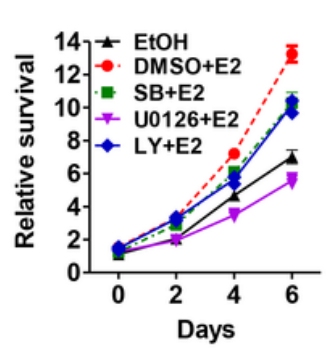

D.

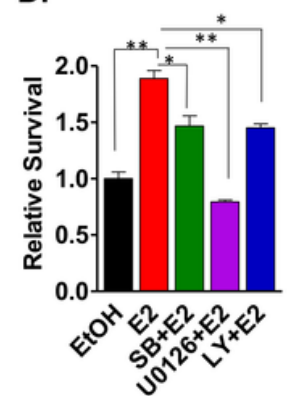

E.

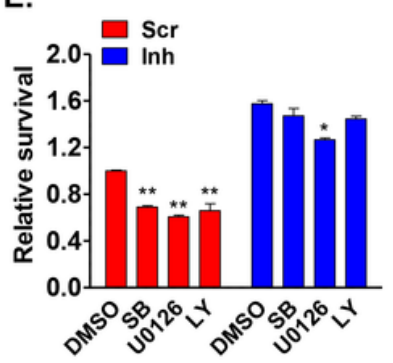

F.

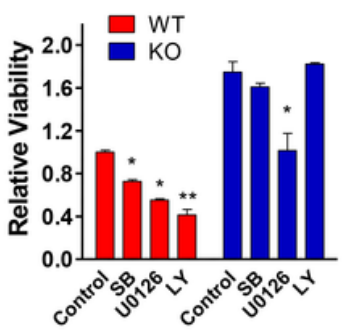

J.

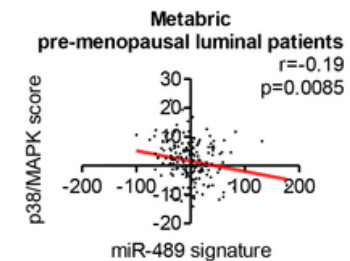

G.
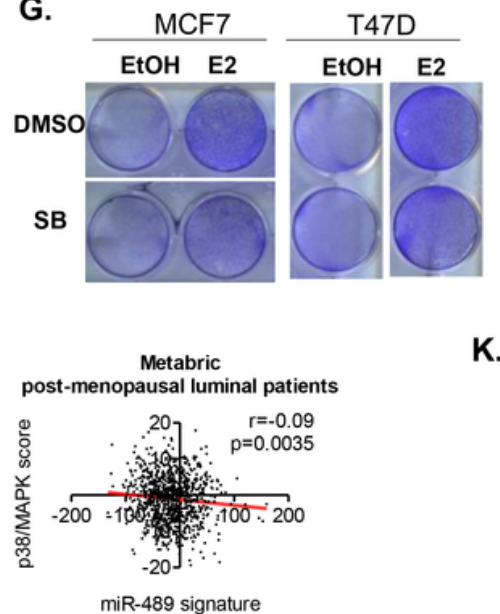

K.
H.
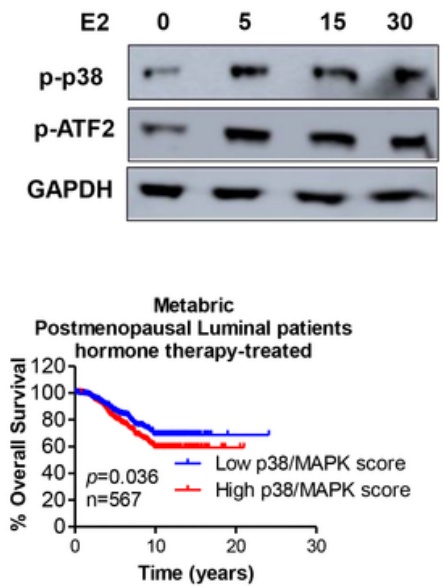

I.

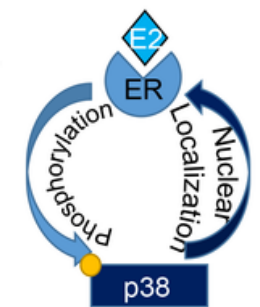

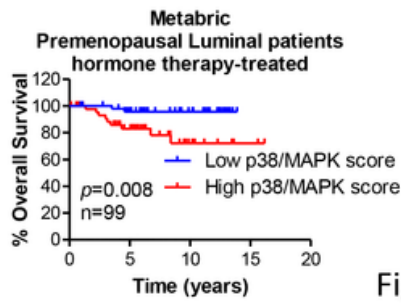

Figure 6

Figure 6

p38 MAPK, PI3K-Akt and MAPK inhibitors phenocopies effect of miR-489 in ER+ breast cancer cell line. A. MCF7 cells were treated with inhibitors of P38 MAPK, ERK and PI3k_AKT pathways and their tole on estrogen induced transcription was examined using luciferase reporter assay. B. MCF7 cells were treated with inhibitors for 24 hours and expression of estrogen responsive genes were examined using qRT-PCR. C-D. MCF7 cells were treated with inhibitors and effect on estrogen induced proliferation was examined using MTT assay. E. MCF7 cells were transfected with scramble or inhibitors and treated with inhibitors for 72 hours in presence of estrogen and effect on proliferation was examined using MTT assay. F. miR489 WT and KO T47D cells were treated with inhibitors in presence of estrogen and cell viability was examined using MTT assay. G. MCF7 cells were treated with DMSO or p38 MAPK inhibitor in presence or absence of estrogen and colony formation assay was performed. H. MCF7 cells were treated with estrogen for indicated time and activation of p38 MAPK signaling was examined using western blot. I. Schematic diagram suggesting positive feedback loop between estrogen signaling and MAPK signaling. J. Correlation of p38 MAPK signature and estradiol signature in pre-and post-menopausal patients. Analysis of patient dataset showing higher p38 MAPK score in pre-menopausal patients compared to post-menopausal patients. K. p38 MAPK signature predicting survival analysis of patients receiving hormone-therapy in pre- and post-menopausal patients. ${ }^{*}, \mathrm{p}<0.05 ;{ }^{*}, \mathrm{p}<0.01 ; * \star *, p<0.001$. Data are means of three replicates \pm SEM.

\section{Supplementary Files}


This is a list of supplementary files associated with this preprint. Click to download.

- SupplementaryData.pdf 